\title{
Simulated evaluation of new switching based median filter for suppressing SPN and RVIN
}

\author{
Vorapoj Patanavijit, Kornkamol Thakulsukanant \\ Assumption University, Thailand
}

\begin{tabular}{l}
\hline \hline Article Info \\
\hline Article history: \\
Received Oct 13, 2018 \\
Revised Feb 15, 2019 \\
Accepted Mar 2, 2019 \\
\hline
\end{tabular}

\section{Keywords:}

AMF (adaptive median filter) Digital image processing NSMF (new switching-based median filtering)

SMF (standard median filtering)

\begin{abstract}
In the past two decades, the SPN (salt and pepper noise) suppressing method is worldwide interested researches on computer vision and image processing hence many SPN suppressing methods have been proposed. In general, the primary goal of SPN removal method is the suppressing of SPN in digital images thereby one of the recent effective and powerful SPN suppressing methods is a new switching-based median filtering (NSMF), which is innovated for suppressing high density SPN. Consequently, this paper thoroughly examines its efficiency and constrain of a new switching-based median filtering when this filter is used for contaminated image, which is synthesized by SPN and RVIN (random-value impulsive noise). In these simulations, six well-known images (Lena, Mobile, Pepper, Pentagon, Girl, Resolution) with two impulsive noise classes (SPN and RVIN) are used for measuring the its efficiency and constrain. An evaluation of the efficiency is conducted with many previous methods in forms of subjective and objective indicators.
\end{abstract}

Copyright $(0) 2019$ Institute of Advanced Engineering and Science. All rights reserved.

\section{Corresponding Author:}

Vorapoj Patanavijit,

Assumption University, Thailand.

Email: Patanavijit@yahoo.com

\section{INTRODUCTION OF NSMF (NEW SWITCHING-BASED MEDIAN FILTERING)}

Digital images [1]-[4] are generally contaminated by impulsive noise [5]-[23] due to communicating unsuccess, improper operating of CCD sensor, ADC synchronized erroneous and memory site erroneous hence noise suppressing method is one of the most vital process for sophisticated digital image process [24]-[26] for instant, face identification, license plate identification, remote sensing, etc. Even through the original Median Filter (SMF) [5]-[7] and Adaptive Median Filter (AMF) [14], [27] are known as the practical noise suppressing method [5]-[23] for SPN, one of the recent effective and powerful SPN suppressing methods is a NSMF (new switching-based median filtering) [28], which is proposed for suppressing only SPN, especially high density. From some results [28], it can conclude that NSMF has good efficiency while the NSMF has low computational complexity however there are no research of the NSMF for SPN at all density and random-value impulsive noise. Consequently, this paper thoroughly examines its efficiency and constrain of a novel modified median filtering based switching technique.

\section{STATISTICAL THEORY OF NSMF}

The NSMF comprises of four modified processes (Process 1- Process 4) as showing in Figure 1 instead of three processes (for previous proposed method), namely, detection, estimation, and replacement.

a) Process 1: Detecting the processed pixel as noisy pixel or noiseless pixel. If the processed pixel is 0 or 255 then the processed pixel is classified as contaminated noise otherwise the pixel is noiseless.

b) Process 2: Substituting the processed input pixel by using 1st order linear predictor.

c) Process 3: Estimating the expected original image by using a median filtering based on L-estimators. 
d) Process 4: Replacing contaminated pixels by the estimated pixels.

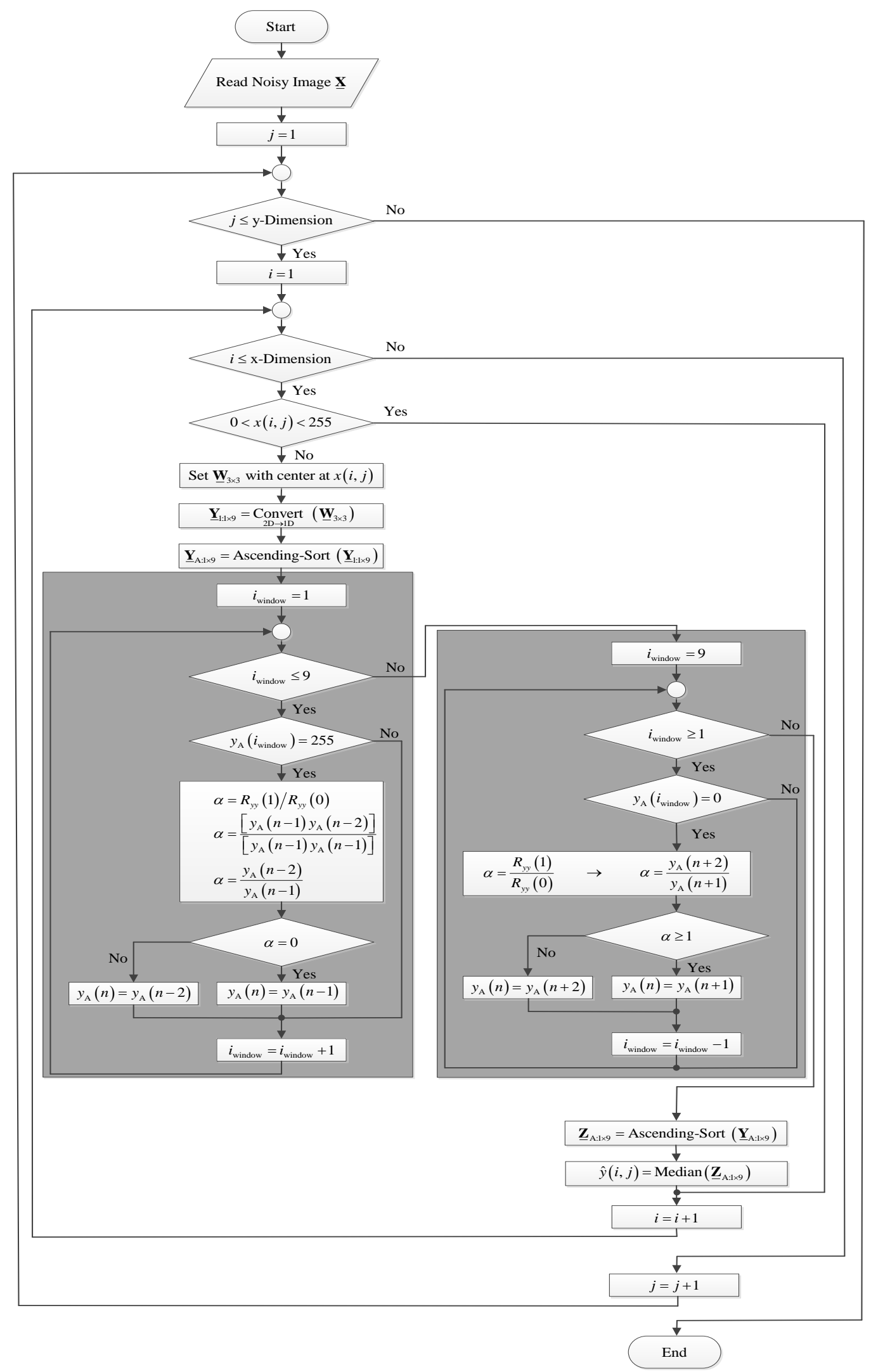

Figure 1. The flowchart of overall method of new switching-based median filtering (NSMF) 


\subsection{Statistical Theory of Estimating Process}

Let $X=\left\{x_{1}, x_{2}, x_{3}, \ldots, x_{j}, x_{j+1}, x_{j+2}, \ldots, x_{n}\right\}$ is the original image, which is noiseless, and $Y=\left\{y_{1}, y_{2}, y_{3}, \ldots, y_{j}, y_{j+1}, y_{j+2}, \ldots, y_{n}\right\}$ is the contaminated image, which is comprised of a set of noiseless pixels $\left\{y_{1}, y_{2}, y_{3}, \ldots, y_{j}\right\}$ and a set of noisy pixels $\left\{y_{j+1}, y_{j+2}, \ldots, y_{n}\right\}$. Let $Z=\left\{y_{1}, y_{2}, y_{3}, \ldots, y_{j}, z_{j+1}, z_{j+2}, \ldots, z_{n}\right\}$, which is comprised of a set of noiseless pixels $\left\{y_{1}, y_{2}, y_{3}, \ldots, y_{j}\right\}$ and a set of substituted pixels for the noisy pixels $\left\{z_{j+1}, z_{j+2}, \ldots, z_{n}\right\}$, and $z_{\text {med }}$ be the median of $Z$.

Let $x_{i}[n]$ is the $i^{\text {th }}$ order statistic of the original image and $\hat{x}[n]$ is the expected original image, which can be defined from set of original noiseless pixels $\left(x_{i}[n]\right)$. By linear prediction, Finite Impulse Response (FIR) linear predictor of order $(p-1)$ can be statistically defined as:

$$
\hat{x}[n+1]=\sum_{k=0}^{P-1} h[n] x[n-k]
$$

where $h[k]$ are the prediction filter coefficients.

The $h[k]$ is statistically defined by the Wiener-Hopf [5] equation as

$$
R_{x}[k] h[k]=r_{x}[k]
$$

where $R_{x}[k]$ is an autocorrelation matrix, $h[k]$ is predictor coefficient vector, and $r_{x}[k]$ is autocorrelation vector. The autocorrelation $R_{x}[k]$ can be statistically defined as

$$
E[x[l-k] x[n-k]]=R_{x}[k-1]
$$

where $k=0$ to $(p-1)$ and $l=0$ to $(p-1)$

By Auto Regressive Moving Average (ARMA) in time domain, the causal Infinite Impulse Response (IIR) predictor is given by $H[z]=z(1-1 / Q[z])$, which can be statistically defined as

$$
\hat{x}[n+1]=\sum_{k=0}^{N-1} a_{k} \hat{x}[n-k]+\sum_{k=0}^{N-1} b_{k} \hat{x}[n-k]
$$

Let $\hat{x}[n]$ is an expected original image from one or more noiseless pixels and $\hat{x}[n]=d[k]$

$$
E[\hat{x}[n] x[n+1]]=E[d[n] x[n+1]]=\hat{r} d[k]
$$

\subsection{Statistical Theory of Replacing Process}

If the processed input pixel is 0 or 255 then the pixel is defined as a noisy pixel and is replace by the replacing process, which comprises of 10 processing step as following:

1) Processing Step 1: Setting the $3 \times 3$ window with center at the processed pixel $x(i, j)$.

2) Processing Step 2: If $0<x(i, j)<255$ then the processed input pixel is classified as noiseless pixel and it is left unchanged and, then, the processed pixel $x(i, j)$ moves to the next position.

3) Processing Step 3: If $x(i, j)=0$ or $x(i, j)=255$ then the processed input pixel is classified as noisy pixel and go to Processing Step 4.

4) Processing Step 4: Converting the $3 \times 3$ window (2D) to the vector $Y_{A}$ (1D)

5) Processing Step 5: Sorting to the vector $Y_{A}$ (1D) in ascending order

6) Processing Step 6: If $x[n]=255$ then replacing $x[n]$ from left to right by following equation 


$$
\begin{aligned}
& x[n]=\alpha \cdot x[n-1] \text { with } \alpha=\left(R_{x x}[1] / R_{x x}[0]\right), 0<\alpha<1 \\
& R_{x x}[1]=x[n-1] \cdot x[n-2] \\
& R_{x x}[0]=x[n-1] \cdot x[n-1] \\
& \text { If } \alpha=0 \text { then } x[n]=x[n-1]
\end{aligned}
$$

a) Processing Step 7: If $x[n]=0$ then replacing $x[n]$ from right to left by following equation

$$
\begin{aligned}
& x[n]=\alpha \cdot x[n+1] \text { with } \alpha=\left(R_{x x}[1] / R_{x x}[0]\right), 0<\alpha<1 \\
& R_{x x}[1]=x[n+1] \cdot x[n+2] \\
& R_{x x}[0]=x[n+1] \cdot x[n+1] \\
& \text { If } \alpha \geq 1 \text { then } x[n]=x[n+1]
\end{aligned}
$$

b) Processing Step 8: Estimating the vector $Z_{A}(1 \mathrm{D})$ by the predicted value, Sort the vector $Z_{A}(1 \mathrm{D})$, and Determine the median value.

c) Processing Step 9: Replace the processed pixel $x(i, j)$ with its median value.

d) Processing Step 10: Reprocess the Processing Steps 1 to Processing Steps 3 until the entire image is processed completely.

\section{ILLUSTRATION OF NSMF}

In this NSMF calculation example, the processed pixel intensity is 255 therefore the processed pixel is noisy and the processed pixel is suppressed by NSMF as shown in Figure 2. From the NSMF process, the denoised pixel is suppressed and the output pixel is replaced to be "200".

\section{SIMULATION OUTCOMES}

In this simulation section under both SPN and RVIN, six tested images (Lena (256x256), Mobile (704x480), Pepper (256x256), Pentagon (512x512), Girl-Tiffany (256x256) and Resolution (128x128)) are employed to analytically simulate the upper bound of NSMF efficiency. This simulation analyses the noise suppressing efficiency of the NSMF by first applying the SPN and the RVIN on tested images. Subsequently, the NSMF processes for suppressing the noisy images, which are used to compute the PSNR with the known original images. From the simulation outcomes in Table 1 for SPN (salt\&pepper noise), the NSMF algorithms have the better quality outcomes than SMF (Standard Median Filter) and GMF (Gaussian Mean Filter) at all cases however the NSMF algorithms have the better quality outcomes than AMF for high noise density.

From the simulation outcomes in Table 2 for RVIN, the NSMF algorithms have the better quality outcomes than SMF (Standard Median Filter), GMF (Gaussian Mean Filter) and AMF at all cases. However, the NSMF algorithms have the worst quality outcomes than AMF for all noise density in Resolution image because this image pixel intensity are " 0 " or " 255 ". 


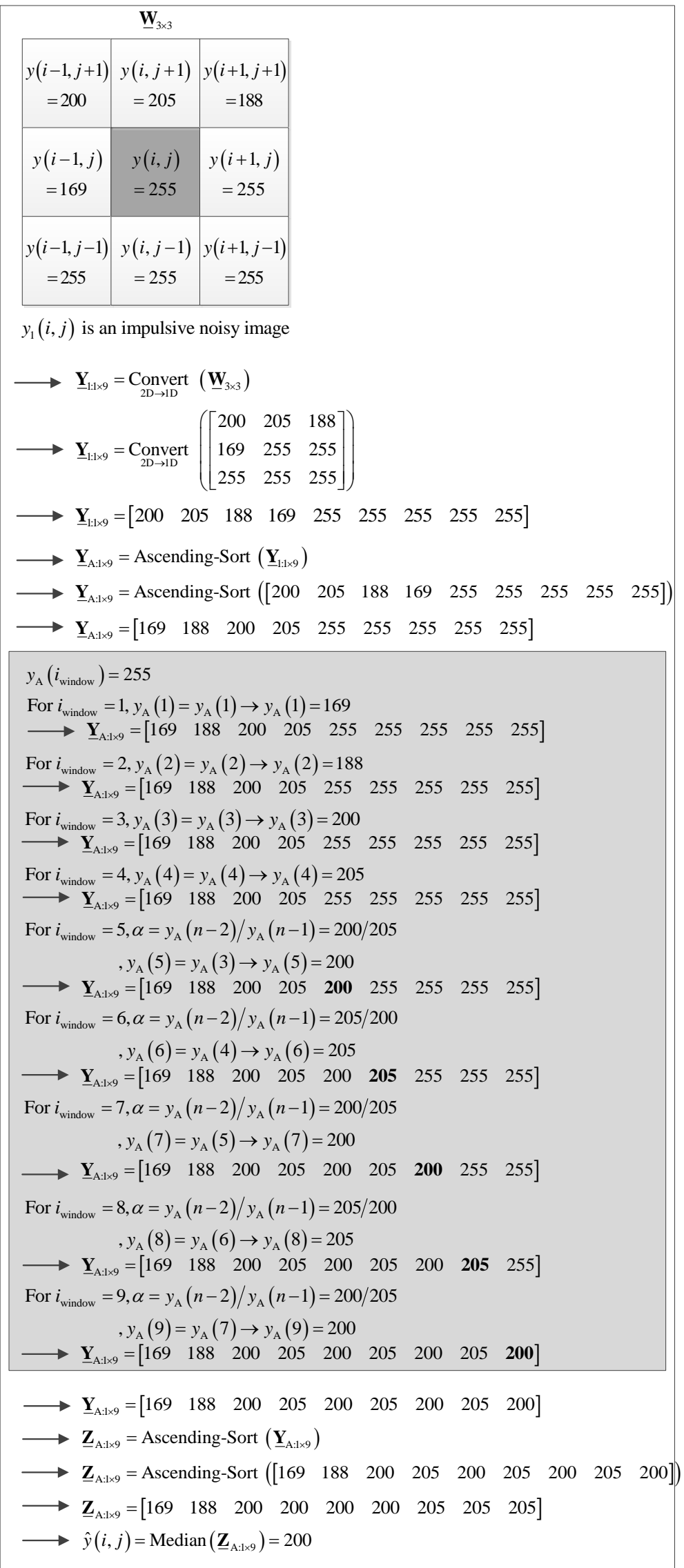

Figure 2. The example of overall calculation of new switching-based median filtering (NSMF) 
Table 1. Denoising Performance Result of SPN

\begin{tabular}{|c|c|c|c|c|c|c|}
\hline SPN & \multicolumn{6}{|c|}{ PSNR (dB) } \\
\hline \multirow{2}{*}{ Tested Images } & \multirow{2}{*}{$\begin{array}{l}\text { Noise } \\
\text { Density }\end{array}$} & \multirow{2}{*}{$\begin{array}{l}\text { Observed } \\
\text { Image }\end{array}$} & \multicolumn{4}{|c|}{ Denoising Algorithm } \\
\hline & & & SMF & GMF & AMF & NSMF \\
\hline \multirow{9}{*}{$\begin{array}{l}\text { Lena } \\
(256 \times 256)\end{array}$} & 10 & 15.6564 & 30.7076 & 19.3812 & 35.3032 & 31.3470 \\
\hline & 20 & 12.6389 & 27.6257 & 16.3208 & 32.1558 & 30.2476 \\
\hline & 30 & 10.8971 & 23.6811 & 14.5829 & 27.9141 & 29.6058 \\
\hline & 40 & 9.6481 & 19.0080 & 13.2479 & 23.7903 & 28.5528 \\
\hline & 50 & 8.6553 & 15.4758 & 12.2146 & 20.5725 & 27.0119 \\
\hline & 60 & 7.7813 & 13.6792 & 11.2939 & 18.4727 & 25.7010 \\
\hline & 70 & 7.1697 & 11.0400 & 10.6509 & 17.0517 & 25.2202 \\
\hline & 80 & 6.5846 & 8.8975 & 10.0057 & 16.5452 & 23.6491 \\
\hline & 90 & 6.0604 & 7.1223 & 9.4356 & 17.1346 & 21.7870 \\
\hline \multirow{9}{*}{$\begin{array}{l}\text { Mobile } \\
(704 \times 480)\end{array}$} & 10 & 15.1637 & 21.3601 & 18.4448 & 25.0280 & 21.5039 \\
\hline & 20 & 12.0780 & 20.1976 & 15.4276 & 23.8172 & 20.8137 \\
\hline & 30 & 10.3465 & 18.3435 & 13.6485 & 21.7980 & 20.1805 \\
\hline & 40 & 9.0919 & 16.1073 & 12.3414 & 19.5320 & 19.6503 \\
\hline & 50 & 8.1216 & 13.4726 & 11.3057 & 17.3015 & 19.0300 \\
\hline & 60 & 7.3397 & 12.6259 & 10.4380 & 15.9416 & $\mathbf{1 7 . 8 3 2 7}$ \\
\hline & 70 & 6.6636 & 10.2402 & 9.6946 & 14.5107 & 16.9717 \\
\hline & 80 & 6.1003 & 8.1954 & 9.0778 & 13.6481 & 16.5924 \\
\hline & 90 & 5.5706 & 6.4911 & 8.4680 & 13.1971 & 15.5046 \\
\hline \multirow{9}{*}{$\begin{array}{l}\text { Pepper } \\
(256 \times 256)\end{array}$} & 10 & 15.3798 & 30.6116 & 19.0677 & 36.0391 & 32.0762 \\
\hline & 20 & 12.3593 & 26.5888 & 15.9804 & 31.6485 & 30.3296 \\
\hline & 30 & 10.6242 & 22.0663 & 14.1748 & 26.7650 & 29.3512 \\
\hline & 40 & 9.3998 & 18.4321 & 12.9076 & 23.4995 & 28.4487 \\
\hline & 50 & 7.9930 & 14.8506 & 11.8117 & 20.2203 & 26.6461 \\
\hline & 60 & 7.6189 & 12.0128 & 10.9563 & 18.3003 & 24.8222 \\
\hline & 70 & 6.9246 & 9.7704 & 10.2039 & 16.8667 & 24.1151 \\
\hline & 80 & 6.3710 & 8.0166 & 9.5853 & 16.3827 & 22.7764 \\
\hline & 90 & 5.8582 & 6.5767 & 9.0214 & 16.7617 & 20.8912 \\
\hline \multirow{9}{*}{$\begin{array}{l}\text { Pentagon } \\
(512 \times 512)\end{array}$} & 10 & 15.7999 & 28.7784 & 19.5038 & 32.8245 & 29.2477 \\
\hline & 20 & 12.7934 & 26.5128 & 16.5198 & 30.5509 & 28.4111 \\
\hline & 30 & 11.0668 & 22.8646 & 14.7790 & 27.1212 & 27.8224 \\
\hline & 40 & 9.8125 & 18.9056 & 13.5122 & 23.3677 & 27.0890 \\
\hline & 50 & 8.8227 & 15.4225 & 12.5108 & 20.4567 & 25.9327 \\
\hline & 60 & 8.0450 & 12.6240 & 11.7138 & 18.3989 & 24.7612 \\
\hline & 70 & 7.3650 & 10.3040 & 10.9953 & 17.0422 & 24.2225 \\
\hline & 80 & 6.7914 & 8.4840 & 10.4099 & 16.4737 & 22.9866 \\
\hline & 90 & 6.2778 & 6.9876 & 9.8698 & 16.8647 & 21.4828 \\
\hline \multirow{9}{*}{$\begin{array}{l}\text { Girl-Tiffany } \\
(256 \times 256)\end{array}$} & 10 & 13.6890 & 31.5583 & 17.2530 & 36.8900 & 32.9954 \\
\hline & 20 & 10.6567 & 25.5153 & 13.9593 & 32.0377 & 30.9499 \\
\hline & 30 & 8.8677 & 20.7738 & 11.9599 & 27.6911 & 29.1071 \\
\hline & 40 & 7.5798 & 16.5146 & 10.4543 & 23.3733 & 28.6836 \\
\hline & 50 & 6.5712 & 13.0319 & 9.2367 & 20.1711 & 27.6182 \\
\hline & 60 & 5.8609 & 10.4981 & 8.3590 & 18.5314 & 26.9544 \\
\hline & 70 & $5.13 ` 11$ & 8.0463 & 7.4271 & 17.1068 & 25.7710 \\
\hline & 80 & 4.5674 & 6.2520 & 6.6881 & 16.7480 & 24.2522 \\
\hline & 90 & 4.0573 & 4.7465 & 5.9986 & 17.1560 & 22.3078 \\
\hline \multirow{9}{*}{$\begin{array}{l}\text { Resolution } \\
(128 \times 128)\end{array}$} & 10 & 13.4819 & 17.9425 & 16.3688 & 18.3302 & 12.9262 \\
\hline & 20 & 10.1271 & 16.2124 & 13.0706 & 17.1273 & 12.8794 \\
\hline & 30 & 8.4430 & 14.4548 & 11.1807 & 15.7907 & 12.4083 \\
\hline & 40 & 7.3308 & 12.6223 & 9.9457 & 14.7904 & 11.6991 \\
\hline & 50 & 6.2938 & 10.4851 & 8.7005 & 13.8816 & 10.6690 \\
\hline & 60 & 5.4436 & 8.5925 & 7.6865 & 14.0392 & 10.2361 \\
\hline & 70 & 4.6795 & 6.6295 & 6.7002 & 12.6150 & 10.4634 \\
\hline & 80 & 4.1940 & 5.3585 & 6.0922 & 10.9746 & 9.7668 \\
\hline & 90 & 3.7113 & 4.2234 & 5.4638 & 9.3116 & 8.4931 \\
\hline
\end{tabular}


Table 2. Denoising Performance Result of RVIN

\begin{tabular}{|c|c|c|c|c|c|c|}
\hline RVIN & \multicolumn{6}{|c|}{ PSNR (dB) } \\
\hline \multirow{2}{*}{$\begin{array}{l}\text { Tested } \\
\text { Images }\end{array}$} & \multirow{2}{*}{$\begin{array}{c}\text { Noise } \\
\text { Density }\end{array}$} & \multirow{2}{*}{$\begin{array}{l}\text { Observed } \\
\text { Image }\end{array}$} & \multicolumn{4}{|c|}{ Denoising Algorithm } \\
\hline & & & SMF & GMF & AMF & NSMF \\
\hline \multirow{9}{*}{$\begin{array}{l}\text { Lena } \\
(256 \times 256)\end{array}$} & 10 & 19.7193 & 31.1555 & 23.2638 & 28.4992 & 31.2960 \\
\hline & 20 & 16.6527 & 29.7106 & 20.1102 & 23.1270 & 29.9009 \\
\hline & 30 & 14.9222 & 27.5271 & 18.2831 & 20.1302 & 28.0652 \\
\hline & 40 & 13.6990 & 24.9693 & 16.9480 & 18.0338 & 27.2951 \\
\hline & 50 & 12.6883 & 22.3406 & 15.8415 & 16.3540 & 25.9716 \\
\hline & 60 & 11.8913 & 19.7498 & 14.9352 & 14.9043 & 24.2453 \\
\hline & 70 & 11.2184 & 17.7591 & 14.1493 & 13.7787 & 22.5265 \\
\hline & 80 & 10.6422 & 16.0345 & 13.4958 & 12.8356 & 20.3198 \\
\hline & 90 & 10.1515 & 14.5334 & 12.9029 & 12.0228 & 17.8884 \\
\hline \multirow{9}{*}{$\begin{array}{l}\text { Mobile } \\
(704 \times 480)\end{array}$} & 10 & 18.4574 & 21.4778 & 21.1512 & 22.6605 & 21.5601 \\
\hline & 20 & 15.5151 & 20.8069 & 18.3393 & 19.7674 & 20.9019 \\
\hline & 30 & 13.7727 & 19.7265 & 16.5214 & 17.4115 & 19.8296 \\
\hline & 40 & 12.5299 & 18.5715 & 15.1796 & 15.6813 & 18.7463 \\
\hline & 50 & 11.5304 & 17.1060 & 14.0526 & 14.1777 & 18.0061 \\
\hline & 60 & 10.7497 & 15.5745 & 13.1263 & 12.9999 & 16.9705 \\
\hline & 70 & 10.0875 & 14.2337 & 12.3371 & 11.9870 & 15.7658 \\
\hline & 80 & 9.4794 & 12.9625 & 11.5980 & 11.0907 & 14.4949 \\
\hline & 90 & 8.9565 & 11.8224 & 10.9422 & 10.2919 & 13.1973 \\
\hline \multirow{9}{*}{$\begin{array}{l}\text { Pepper } \\
(256 \times 256)\end{array}$} & 10 & 19.1143 & 31.4270 & 22.6205 & 27.4518 & 31.9058 \\
\hline & 20 & 16.0921 & 28.8665 & 19.4820 & 22.3782 & 29.7204 \\
\hline & 30 & 14.3745 & 26.5900 & 17.6137 & 19.4227 & 27.4882 \\
\hline & 40 & 13.1825 & 23.3362 & 16.2549 & 17.2699 & 26.3846 \\
\hline & 50 & 12.2029 & 20.7731 & 15.1438 & 15.6064 & 24.5452 \\
\hline & 60 & 11.3328 & 18.2128 & 14.0998 & 14.0825 & 22.4280 \\
\hline & 70 & 10.7068 & 16.2565 & 13.3352 & 13.0203 & 20.1256 \\
\hline & 80 & 10.1086 & 14.5768 & 12.5873 & 12.0629 & $\mathbf{1 7 . 5 4 7 7}$ \\
\hline & 90 & 9.6144 & 13.2495 & 11.9859 & 11.2712 & 15.6818 \\
\hline \multirow{9}{*}{$\begin{array}{l}\text { Pentagon } \\
(512 \times 512)\end{array}$} & 10 & 20.2113 & 29.1520 & 23.6997 & 28.2423 & 29.2203 \\
\hline & 20 & 17.2386 & 28.0433 & 20.7616 & 23.8125 & 28.2529 \\
\hline & 30 & 15.4355 & 26.6678 & 18.9263 & 20.7887 & 26.9234 \\
\hline & 40 & 14.1860 & 24.9238 & 17.6449 & 18.7135 & 26.0936 \\
\hline & 50 & 13.2544 & 22.9472 & 16.6548 & 17.1075 & 25.3502 \\
\hline & 60 & 12.4342 & 20.9049 & 15.7838 & 15.7400 & 24.1241 \\
\hline & 70 & 11.7829 & 19.0652 & 15.0817 & 14.6359 & 23.1046 \\
\hline & 80 & 11.1849 & 17.3449 & 14.4326 & 13.6558 & 21.7975 \\
\hline & 90 & 10.6746 & 15.8671 & 13.8673 & 12.8241 & 20.4014 \\
\hline \multirow{9}{*}{$\begin{array}{l}\text { Girl-Tiffany } \\
(256 \times 256)\end{array}$} & 10 & 16.4414 & 31.6049 & 19.9110 & 25.1339 & 28.3519 \\
\hline & 20 & 13.4343 & 28.1774 & 16.5639 & 19.5720 & 28.4094 \\
\hline & 30 & 11.6674 & 23.8175 & 14.5342 & 16.4549 & 28.0443 \\
\hline & 40 & 10.3946 & 19.8213 & 12.9626 & 14.1248 & 26.7023 \\
\hline & 50 & 9.4483 & 16.7201 & 11.7613 & 12.3869 & 24.4072 \\
\hline & 60 & 8.6223 & 14.0847 & 10.6637 & 10.9357 & 20.5217 \\
\hline & 70 & 7.9734 & 12.1107 & 9.8004 & 9.8160 & 16.6627 \\
\hline & 80 & 7.3939 & 10.4710 & 8.9936 & 8.7676 & 13.1380 \\
\hline & 90 & 6.8638 & 9.1560 & 8.2609 & 7.9151 & 10.6609 \\
\hline \multirow{9}{*}{$\begin{array}{l}\text { Resolution } \\
(128 \times 128)\end{array}$} & 10 & 17.7992 & 18.6254 & 20.1134 & 18.3074 & 10.6040 \\
\hline & 20 & 14.6190 & 17.9190 & 17.1729 & 17.4171 & 11.3422 \\
\hline & 30 & 12.7370 & 17.1231 & 15.3050 & 16.7349 & 11.3862 \\
\hline & 40 & 11.3691 & 16.2456 & 13.8148 & 15.5071 & 11.7564 \\
\hline & 50 & 10.5048 & 15.5229 & 12.8678 & 14.4871 & 11.8203 \\
\hline & 60 & 9.7510 & 14.3607 & 11.9178 & 13.3621 & 12.0505 \\
\hline & 70 & 9.1026 & 13.6671 & 11.1682 & 12.3620 & 12.2006 \\
\hline & 80 & 8.4955 & 12.3904 & 10.4038 & 11.1211 & 12.4048 \\
\hline & 90 & 8.0315 & 11.6735 & 9.8152 & 10.2316 & 12.3036 \\
\hline
\end{tabular}




\section{CONCLUSION}

This in-depth research assesses the efficiency of the noise suppressed method based on NSMF under two impulsive noise classes (SPN and RVIN). These simulations employ on six well-known images (Lena, Mobile, Pepper, Pentagon, Girl, Resolution) under two impulsive noise classes for assessing the highest suppressed images in term of PSNR. Many previous noise suppressed methods, such as SMF (Standard Median Filter), GMF (Gaussian Mean Filter) and AMF, are used to assess the analogy efficiency. From simulation outcomes, the NSMF has a good PSNR for high noise density and this filter can work well for RVIN.

\section{ACKNOWLEDGEMENTS}

The research project was funded by Assumption University.

\section{REFERENCES}

[1] I. Pitas and A. N. Venetsanopoulos, Nonlinear Digital Filters Principles and Applications, Kluwer Academic Publishers, Norwell, Mass, USA, 1990.

[2] J. Astola and P. Kuosmanen, Fundamentals of Nonlinear Digital Filtering, CRC Press, Boca Raton, Fla, USA, 1997.

[3] R. C. Gonzalez and R. E. Woods, Digital Image Processing, Prentice-Hall,Upper Saddle River,NJ, USA, 2nd edition, 2002.

[4] M. H. Hayes, Statistical Digital Signal Processing and Modeling, JohnWiley \& Sons, Singapore, 2002.

[5] W. K. Pratt, "Median filtering," Tech. Rep., Image Proc. Inst., Univ. Southern California, Los Angeles, Sep. 1975.

[6] N. C. Gallagher Jr. and G. L.Wise, "A theoretical analysis of the properties of median filters," IEEE Transactions on Acoustics, Speech, and Signal Processing, vol. 29, no. 6, pp. 1136-1141, 1981.

[7] T. A. Nodes and N. C. Gallagher Jr., "Median filters: some modifications and their properties," IEEE Transactions on Acoustics, Speech, and Signal Processing, vol. 30, no. 5, pp. 739- 746, 1982.

[8] E. Abreu, M. Lightstone, S. K. Mitra, and K. Arakawa, "A new efficient approach for the removal of impulse noise from highly corrupted images," IEEE Transactions on Image Processing, vol. 5, no. 6, pp. 1012-1025, 1996.

[9] D. R. K. Brownrigg, "The weighted median filter," Communications of the ACM, vol. 27, no. 8, pp. 807-818, 1984.

[10] O. Yli-Harja, J. Astola, and Y. Neuvo, "Analysis of the properties of median and weighted median filters using threshold logic and stack filter representation," IEEE Transactions on Signal Processing, vol. 39, no. 2, pp. 395410, 1991.

[11] G. R. Arce and J. L. Paredes, "Recursive weighted median filters admitting negative weights and their optimization," IEEE Transactions on Signal Processing, vol. 48, no. 3, pp. 768-779, 2000.

[12] Y. Dong and S. Xu, "A new directional weighted median filter for removal of random-valued impulse noise," IEEE Signal Processing Letters, vol. 14, no. 3, pp. 193-196, 2007.

[13] T. Chen, K.-K.Ma, and L.-H. Chen, “Tri-state median filter for image denoising," IEEE Transactions on Image Processing, vol. 8, no. 12, pp. 1834-1838, 1999.

[14] H. Hwang and R. A. Haddad, "Adaptive median filters: new algorithms and results," IEEE Transactions on Image Processing, vol. 4, no. 4, pp. 499-502, 1995.

[15] S. Zhang and M. A. Karim, “A new impulse detector for switching median filters," IEEE Signal Processing Letters, vol. 9, no. 11, pp. 360-363, 2002.

[16] H.-L. Eng and K.-K. Ma, "Noise adaptive soft-switching median filter," IEEE Transactions on Image Processing, vol. 10, no. 2, pp. 242-251, 2001.

[17] Z. Wang and D. Zhang, "Progressive switching median filter for the removal of impulse noise from highly corrupted images," IEEE Transactions on Circuits and Systems II, vol. 46, no. 1, pp. 78-80, 1999.

[18] P.-E. Ng and K.-K.Ma, "A switching median filter with boundary discriminative noise detection for extremely corrupted images," IEEE Transactions on Image Processing, vol. 15, no. 6, pp. 1506-1516, 2006.

[19] R. H. Chan, C.-W. Ho, and M. Nikolova, "Salt-and-pepper noise removal by median-type noise detectors and detailpreserving regularization," IEEE Transactions on Image Processing, vol. 14, no. 10, pp. 1479-1485, 2005.

[20] K. S. Srinivasan and D. Ebenezer, "A new fast and efficient decision-based algorithm for removal of high-density impulse noises,” IEEE Signal Processing Letters, vol. 14, no. 3, pp. 189-192, 2007.

[21] S. Schulte, M. Nachtegael, V. DeWitte, D. van der Weken, and E. E. Kerre, "A fuzzy impulse noise detection and reduction method," IEEE Transactions on Image Processing, vol. 15, no. 5, pp. 1153-1162, 2006.

[22] A. Ben Hamza and H. Krim, "Image denoising: a nonlinear robust statistical approach," IEEE Transactions on Signal Processing, vol. 49, no. 12, pp. 3045-3054, 2001.

[23] Z. Wang, A. C. Bovik, H. R. Sheikh, and E. P. Simoncelli, "Image quality assessment: from error visibility to structural similarity," IEEE Transactions on Image Processing, vol. 13, no. 4, pp. 600-612, 2004.

[24] E. Pavankumar, Manojkumar Rajgopal, Robust Visual Multi-Target Trackers: A Review, Indonesian Journal of Electrical Engineering and Computer Science, Vol. 12, No. 1, October 2018, pp. 7-16 
[25] Nur Ateqah Binti Mat Kasim, Nur Hidayah Binti Abd Rahman, Zaidah Ibrahim, Nur Nabilah Abu Mangshor, Celebrity Face Recognition using Deep Learning, Indonesian Journal of Electrical Engineering and Computer Science, Vol. 12, No. 2, November 2018, pp. 476-481

[26] Raden Arief Setyawan, Rudy Sunoko, Mochammad Agus Choiron, Panca Mudji Rahardjo, Implementation of Stereo Vision Semi-Global Block Matching Methods for Distance Measurement, Indonesian Journal of Electrical Engineering and Computer Science, Vol. 12, No. 2, November 2018, pp. 585-591

[27] Vorapoj Patanavijit, Performance Analysis of Denoising Algorithm Based on Adaptive Median Filter Under Unsystematic Intensity Impulse and Salt\&Pepper Noise, The 6th International Electrical Engineering Congress (iEECON2017), Krabi, Thailand, March 2018.

[28] V. Jayaraj and D. Ebenezer, A New Switching-BasedMedian Filtering Scheme and Algorithm for Removal of High-Density Salt and Pepper Noise in Images, EURASIP Journal on Advances in Signal Processing, Hindawi Publishing Corporation, 2010

\section{BIOGRAPHIES OF AUTHORS}

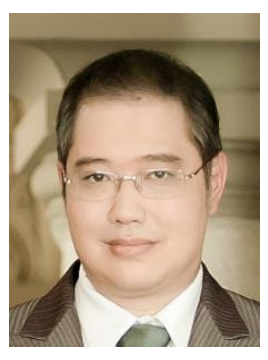

Vorapoj Patanavijit received the B.Eng., M.Eng. and Ph.D. degrees from the Department of Electrical Engineering at the Chulalongkorn University, Bangkok, Thailand, in 1994, 1997 and 2007 respectively. He has served as a full-time lecturer at Department of Electrical and Electronic Engineering, Faculty of Engineering, Assumption University since 1998 where he is currently an Associate Professor. He has authored and co-authored over 150 national/international peerreviewed publications in Digital Signal Processing (DSP) and Digital Image Processing (DIP). He received the best paper awards from many conferences such as ISCIT2006, NCIT2008, EECON33 (2010), EECON-34 (2011), EECON-35 (2012) and etc. Moreover, he is invited to be the guest speaker at IWAIT2014 and contributed the invited paper at iEECON 2014. He has served as a Technical Program Committees (TPC) on Signal Processing of ECTI (Electrical Engineering/Electronics, Computer, Telecommunications and Information Technology) Association, Thailand since 2012 to 2015 . As a technical reviewer of international journals since 2006, he has been assigned to review over 60 journal papers (indexed by ISI) from IEEE Transactions on Image Processing, IEEE Journal of Selected Topics in Signal Processing (JSTSP), IET in Image Processing (IEEE), IEEE Signal Processing Letters (IEEE), EURASIP Journal on Applied Signal Processing (JASP), Digital Signal Processing (Elsevier Ltd.), Journals of Neurocomputing (Elsevier Ltd.), Neural Networks (Elsevier Ltd.), International Journal for Light and Electron Optics (Optik) (Elsevier Ltd.), The Visual Computer (Springer), Journal of Electronic Imaging (SPIE), Journal of Optical Engineering (SPIE), IEICE Journal Electronics Express (ELEX) and ECTI Transactions on CIT (ECTI Thailand). As a technical reviewer of over 40 international/national conferences since 2006, he has been assigned to review over 130 proceeding papers. He has participated in more than 8 projects and research programmed funded by public and private organizations. He works in the field of signal processing and multidimensional signal processing, specializing, in particular, on Image/Video Reconstruction, SRR (Super-Resolution Reconstruction), Compressive Sensing, Enhancement, Fusion, Digital Filtering, Denoising, Inverse Problems, Motion Estimation, Optical Flow Estimation and Registration.

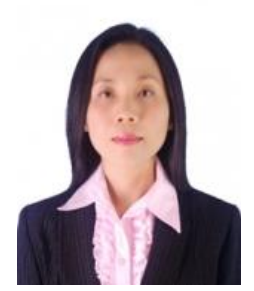

Kornkamol Thakulsukanant received the B.Eng. (Electrical Engineering) from Assumption University, Thailand in 1994, MSc. (Telecommunications and Computer Network Engineering) from London South Bank University, United Kingdom in 1997 and Ph.D. (in Electronic and Electrical Engineering) from Bristol University, United Kingdom in 2009 respectively. She served as a full-time lecturer at Faculty of Science and Technology, Assumption University since 1998 until 2014 and she has served as full-time lecturer at School of Management and Economics, Assumption University where she has been currently an Assistance Professor (in IT) since 2014. She works in the field of Digital Signal Processing (DSP) and Digital Image Processing (DIP), specializing, in particular, on Digital Image Reconstruction/Enhancement. 